\title{
Apropos: Nachhaltigkeit - eine Videoreihe rund um Konsum
}

\author{
*mstandl.bwib-m2019@fh-salzburg.ac.at, Fachhochschule Salzburg, ( $\triangle$ korresp. Autor) \\ **hweinberger.bwib-m2019@fh-salzburg.ac.at, Fachhochschule Salzburg \\ ${ }^{* * *}$ kzenisek.bwib-m2019@fh-salzburg.ac.at, Fachhochschule Salzburg \\ **** cnemedi.bwib-m2019@fh-salzburg.ac.at, Fachhochschule Salzburg
}

eingereicht am: 14.04.2021, akzeptiert am: 27.04.2021

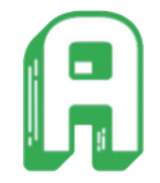

APROPOS

Im vorliegenden Beitrag stellen Studierende der Fachhochschule Salzburg Ergebnisse eines Projekts vor, im Rahmen dessen sie zielgruppenorientierte Videos zum Thema Konsum erstellt haben. Als Zielgruppe werden Jugendliche ab 14 Jahren sowie Lehrkräfte, welche dieses Material im Unterricht verwenden möchten, angesprochen. In diesen Videos werden die Schwerpunkte Overshoot Day, Konsumgeschichte, Konsumpsychologie, Nachhaltigkeit, Sustainable Development Goals, nachhaltiger Konsum und Tipps für einen bewussteren Konsum dargestellt.

Keywords: Nachhaltigkeit, Konsum, Lernvideos, Tipps, Aufklärung, Studierendenprojekt

\section{Wie kam es zum Projekt?}

Im Rahmen der Lehrveranstaltung „Student Studies“ an der Fachhochschule Salzburg wird Studierenden des Masterstudiengangs „Betriebswirtschaft“ ein Freiraum gegeben, wissenschaftlich Themen zu bearbeiten, für die sie sich im Besonderen interessieren und die durch das jeweilige Curriculum nicht abgedeckt werden. Vier berufsbegleitende Masterstudierende der Betriebswirtschaft entschieden, die für die Lehrveranstaltung vorgesehene Zeit für die Auseinandersetzung mit dem Thema „Nachhaltiges Wirtschaften“ zu investieren. Nachdem das Thema sehr umfangreich erschien, wurde es eingegrenzt. Die konkrete Zielsetzung und der Arbeitsbereich wurden eingeschränkt. Es wurde entschieden, den Fokus auf die Umstände in Bezug auf den Ressourcenverbrauch innerhalb Österreichs zu legen, wofür der Projekttitel „Österreich, dein Konsum" passend erschien. Die Einschränkung auf diesen Bereich erschien deshalb so interessant zu sein, da Österreich EU-weit einen hohen Konsum pro Kopf vorweist. Die globale Perspektive sollte das Bild in Relation auf die restliche Welt setzen. Es wurden hier 1000 Stunden investiert, um das Thema Nachhaltigkeit wissenschaftlich zu bearbeiten und Videos zu erstellen, welche zur Sensibilisierung des Themas „nachhaltiger Konsum“ bei Jugendlichen beitragen sollen. Ergebnis dieses Projekts sind ein Bericht und insgesamt acht Videos zu den Themen Overshoot Day (14 min.), Konsumgeschichte (21 min.), Konsumpsychologie (16 min.), Nachhaltigkeit (10 min.), Sustainable Development Goals (7 min.), nachhaltiger Konsum (10 min.) und Tipps für einen bewussteren Konsum (6 min.). Der Erstellung der Videos ging eine umfangreiche wissenschaftliche Literaturrecherche zu den thematisierten Inhalten und Zusammenhängen voraus. Die Ergebnisse wurden in einem wissenschaftlichen Bericht zusammengefasst. Gewählt wurden diese Themenbereiche nach den jeweiligen Interessenslagen der Projektmitglieder, welche tiefere Einblicke in die Themen gewinnen wollten. Dabei wurde allerdings ebenso auf ein Gesamtbild wert gelegt, indem die wissenschaftliche Diskussion und konträre Meinungen dazu recherchiert und verglichen wurden. Betreut wurde dieses Projekt von Ing. Mag. Andreas Griebel, MA, welcher die Autoren dieses Beitrags jederzeit unterstützte und zur Reflexion anregte.

\section{Zielgruppe}

Die Zielgruppe der Videoreihe sind vor allem Jugendliche ab 14 Jahren. Ab diesem Alter wird gesetzlich von mündigen Minderjährigen ausgegangen, welche ${ }^{*}$ eine beschränkte (aber doch keine erweiterte) Geschäftsfähigkeit hat. Ab diesem Alter sind Jugendliche 
durch diese Tatsache verstärkt in das Konsumleben eingebunden. Ihre Handlungsfähigkeit soll durch Wissen weiterentwickelt werden. Darüber hinaus gilt es, die Vernetzung zwischen Wirtschaft, Gesellschaft und der Umwelt hervorzuheben und der jüngeren Generation bewusst zu machen. Die Videoreihe kann im formellen als auch im nicht-formellen Unterricht zur Sensibilisierung eingesetzt werden. Zudem bilden in Zeiten des Distance Learnings Lernvideos praktikable Unterstützungsformate. Darüber hinaus kann jede ${ }^{*}$ Interessierte sich durch die Videoreihe klicken, um Neues zu erfahren.

\section{Methodik}

Der Fokus lag auf fachlicher Klärung und Aktualität der Quellen. Die kritische Gegenüberstellung verschiedener Aussagen war ein wesentlicher Fokus bei der Erstellung, um keine einseitige Darstellung zu vermitteln. Bei der anschließenden Erstellung der Videoreihe war es eine große Herausforderung, den beiden Anforderungsgruppen gerecht zu werden. Einerseits musste die Arbeit den wissenschaftlichen Standards entsprechen und anderseits mussten die daraus entstanden Videos für die Zielgruppe verständlich sein. Somit transformierten die Verfasser, nach Fertigstellung der wissenschaftlichen Abhandlung, den Text in eine für Jugendliche verständlichere Sprache. Ebenso musste ein ansprechendes Format gefunden werden, um die Inhalte der Zielgruppe zu präsentieren. Es wurden ansprechende Animationsvideos mit der Software "Toonly" erstellt, welche mit Sprache hinterlegt wurden. Zusätzlich wurden Diagramme und Graphiken selbst erstellt sowie recherchiert, um die Inhalte noch möglichst anschaulich der Zielgruppe näherzubringen. Das gewählte Transfermedium hat sich aus mehreren Gründen bewährt. Die Hauptfigur der Videoserie heißt "Günther" und führt die Seher*innen durch das Programm, erklärt Sachverhalte und regt die Aufmerksamkeit an. Günther ist in Abb. 1 dargestellt. Um die Aufmerksamkeit der Seher*innen zusätzlich zu erhöhen, wurden Teile des Videos an geeigneten

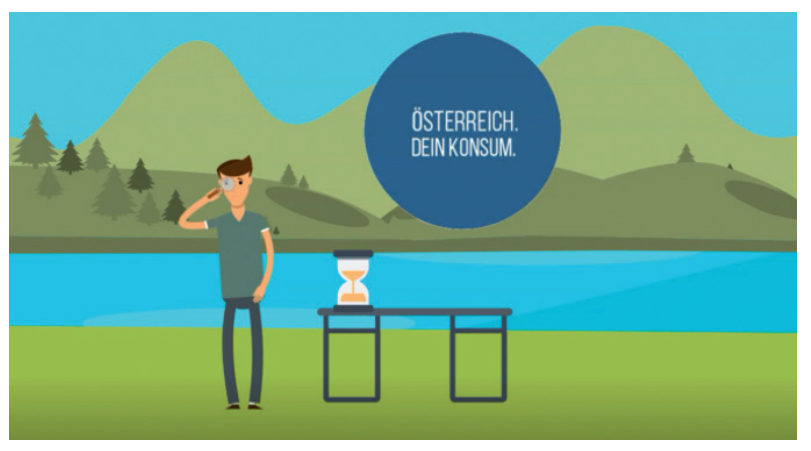

Abb. 1: Ein Screenshot aus einem Erklärvideo mit der Hauptfgur „Günther“
Stellen in mehrere Teile unterteilt, um Cliffhanger ${ }^{1}$ aufzubauen und die Neugierde auf Mehr anzuregen. Darüber hinaus macht das Medium Video es möglich, individuell Pausen einzulegen und bestimmte Stellen nochmal anzusehen. Zudem war es ein wesentliches Anliegen, die Videos über eine frei zugängliche Videoplattform allgemein zugänglich zu machen.

\section{Ziel}

Die Hintergründe zum Start des Projekts waren persönlicher Natur, denn die intrinsische Motivation eines jeden einzelnen Projektmitglieds hat dazu beigetragen, dem dringlichsten Ziel vor allem gerecht zu werden: Die Entwicklung der Fähigkeiten von bewussten und kritischen Konsument*innen soll verstärkt werden, indem wichtige Eckpfeiler näher erläutert und dargestellt werden. Gerade jüngere Generationen sollen auf wissenschaftlichem Niveau im Sinne einer hochschulischen Transferleistung erreicht werden. Dementsprechend wurde das Ziel verfolgt, ein Komplexitätsniveau zu wahren, welches nicht überfordert und dabei der hohen Komplexität des Themas gerecht wird. Letztlich war die Erstellung einer Videoreihe im Sinne eines nachhaltigen Transfers der Forschungsergebnisse - im Fokus. Hierbei wurde maßgebliches Augenmerk auf die Erstellung von Medien zur Kommunikation der Erkenntnisse gelegt. Darüber hinaus war es jedem einzelnen Projektmitglied wichtig, vor dem Hintergrund einer betriebswirtschaftlichen Ausbildung über den Tellerrand zu blicken, Neues zu entdecken und zu lernen. Der Freiraum im Curriculum sollte dadurch bestmöglich genutzt werden, um mit Leidenschaft, Kreativität und Spaß an einem Thema allgemeinen Interesses zu arbeiten. Ein großes Anliegen ist es, die Zielgruppe zur Reflexion anzuregen. Wir möchten gerne helfen, den Gedanken zu verbreiten bzw. zu verstärken, dass jedes Individuum durch die Haltung „Bewusster Konsum“ einen Beitrag zu einer nachhaltigen Entwicklung leisten kann. Wir hoffen und würden uns darüber freuen, wenn die von uns erstellte Videoreihe mit ihren vielfältigen Einbettungsmöglichkeiten im GW-Unterricht einen Teil dazu beitragen kann.

\section{Offen für Feedback}

Als Studierende der Betriebswirtschaft haben sich die Projektmitglieder intensiv mit dem Thema nachhaltiger

Cliffhanger werden hauptsächlich als ein offener Ausgang einer Episode auf ihrem Höhepunkt mit z. B. Fernsehserien oder seltener mit planvoll fortgesetzten Kinofilmen assoziiert. DieHandlungwird meist in der nächsten oder einer noch späteren Episode fortgesetzt. 
Konsum beschäftigt und die Ergebnisse in zielgruppenorientierte Videos für Jugendliche überführt. Sollten sich die Videos in die Gestaltung des Unterrichts berücksichtigen lassen, würden sich die Ersteller über Verbesserungsvorschläge und kritische Reflexion freuen.

Sollten Sie an einer fortlaufenden Zusammenarbeit interessiert sein oder eventuell sogar ganz andere Themen mit uns besprechen wollen, dann zögern Sie bitte nicht. Das Projektteam steht gerne für den Austausch zur Verfügung.

Richten Sie bitte Kritik bzw. Feedback und Anfragen an Moritz Standl unter folgender E-Mail-Adresse: moritz.apropos@gmail.com

\section{Videos auffindbar wo?}

Zum Ende unseres Beitrags möchten wir abschließend noch auf unsere Lernvideos verweisen. Zur besseren Verbreitung wurde ein YouTube-Kanal eröffnet. Sie finden diesen Kanal unter dem QR-Code oder unter folgenden

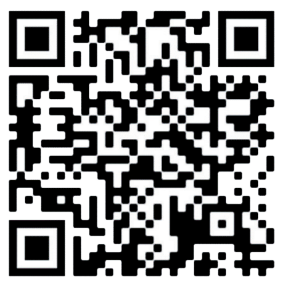
Link:

https://www.youtube.com/channel/UCpUFismjN5JcCzpvbANcX8w/featured 